\title{
USE OF A LARGE MULTICELL IONIZATION DETECTOR - THE EXTERNAL PARTICLE IDENTIFIER - IN EXPERIMENTS WITH THE BEBC HYDROGEN BUBBLE CHAMBER
}

\author{
V. BARUZZI ${ }^{4}$, R. CAROSIO ${ }^{5}$, F. CRIJNS ${ }^{6}$, L. GERDYUKOV ${ }^{7}$, Y. GOLDSCHMIDT- \\ CLERMONT $^{4}$, A. GRANT ${ }^{4}$, D. JOHNSON ${ }^{3}$, F. KRÖNER ${ }^{9}$, I. LEHRAUS ${ }^{4}$, \\ R. MATTHEWSON ${ }^{4}$, C. MILSTENE ${ }^{8}$, V. NIKOLAENKO ${ }^{7}$, Y. OREN $^{8}$, Y. PETROVIKH ${ }^{7}$, \\ R.T. ROSS ${ }^{4}$, P. SIXEL ${ }^{1}$, M. SPYROPOULOU-STASSINAKI ${ }^{2}$, A. STERGIOU ${ }^{6}$, W. TEJESSY ${ }^{4}$, \\ P. THEOCHAROPOULOS ${ }^{3}$, G. VASSILIADIS ${ }^{2}$, P.R.S. WRIGHT ${ }^{4}$ and J. ZOLL ${ }^{4}$ \\ ${ }^{\prime}$ III. Physikalisches Inst. der Techn. Hochschule, Aachen, Germany \\ ${ }^{2}$ Nuclear Physics Laboratory, University of Athens, Athens, Greece \\ ${ }^{3}$ Inter-University Institute for High Energies, Brussels, Belgium \\ ${ }^{4}$ CERN, European Organization for Nuclear Research, Geneva, Switzerland \\ 'Sezione INFN and Istituto di Scienze Fisiche, Genova, Italy \\ ${ }^{\circ}$ University of Nijmegen, Nijmegen, The Netherlands \\ 'Institute for High Energy Physics, Serpukhov, U.S.S.R. ** \\ ${ }^{8}$ Department of Physics and Astronomy, Tel-Aviv University, Tel-Aviv, Israel \\ ${ }^{9}$ Institut für Hochenergiephysik der Österreichischen Akademie der Wissenschaften, Vienna, Austria
}

Received 9 September 1982

Fast secondary particles from interactions of $\mathrm{K}^{+}$at $70 \mathrm{GeV} / \mathrm{c}$ and $\mathrm{K}^{-}$at $110 \mathrm{GeV} / \mathrm{c}$ in the $\mathrm{BEBC}$ hydrogen bubble chamber are identified by use of the relativistic rise of ionization in argon. The ionization is measured in a large multicell ionization counter system. the External Particle Identifier (EPI). The operation of this hybrid system, the calibration and the procedures of off-line analysis leading to particle identification are described. For a sample of 9283 secondary particles collected in the $\mathrm{K}^{+} \mathrm{p}$ experiment, we present the measurements of ionization, and the determination of acceptance and identification efficiencies. The momentum-dependent populations of pions, kaons and protons have been obtained. Track by track identification procedures yielding samples of pions and kaons with a contamination $\leqslant 12 \%$ are discussed. A study of the resolution of the EPI in these experimental conditions shows average values in a range of $7.6 \%$ to $11 \%$ depending on the number of ionization samples remaining after removal of background. These values extrapolate to a value consistent with the $6.6 \%$ fwhm measured over the whole device for single particles during calibration with a 50 $\mathrm{GeV} / c \pi^{-}$beam.

\section{Introduction}

In order to identify particles over a wide range of momenta, the hydrogen bubble chamber BEBC has been equipped with a multicell proportional chamber device known as the EPI (External Particle Identifier). This detector samples the ionization, in the traversed gas, of fast outgoing particles - up to a maximum of 128 samples per track - in order to exploit the relativistic rise of ionization. The measurement of ionization obtained from these samples gives a value for the particle velocity. This, combined with the measurement of

* Now at Olivetti S.A., Ivrea, Italy.

** Participating under the terms of the 1967 agreement between CERN and the USSR State Committee for the Utilization for Atomic Energy. momentum in BEBC, enables the determination of the mass of the fast particles emitted in a forward cone limited by the exit window of the bubble chamber.

Design and construction of the EPI as well as preliminary tests have been described previously [ $1-4]$. The relativistic rise of ionization in the chosen gas ( $\mathrm{Ar}+5 \%$ $\mathrm{CH}_{4}$ ) and the achieved resolution limits are given in ref. 5 , as obtained from tests with the full EPI placed in the hadron beam leading to $\mathrm{BEBC}$.

Here, we describe the operation of the EPI as part of a hybrid system comprising the rf separated beam, a Cherenkov counter tagging system for the incoming particles, the $3.7 \mathrm{~m}$ hydrogen bubble chamber BEBC, downstream wire chambers and the EPI. Two hadron experiments using this hybrid system were carried out with incident $110 \mathrm{GeV} / c \mathrm{~K}^{-}$[6] and $70 \mathrm{GeV} / c \mathrm{~K}^{+}$[7]. The use of the EPI in these experiments required the development of various techniques, such as association 
of the data from the different components of the hybrid system, pattern recognition and ionization analysis methods. The results of these technical studies and the level of particle separation achieved are given in the present paper. Some of the techniques have been presented in preliminary form in ref. 8 . Studies of the physics of $\mathrm{K}^{ \pm} \mathrm{p}$ interactions using the EPI are in progress and preliminary results were presented [9].

The paper is organized as follows: sect. 2 presents the hybrid system; it summarizes the EPI design, describes the EPI wire chambers, discusses the on-line data acquisition, control and monitoring system and gives an account of the calibration and data taking runs. Sect. 3 is devoted to the offline software: association of the bubble chamber and EPI information, pattern recognition of the tracks in the EPI, removal of background (cleaning), measurement of ionization and particle identification. Sect. 4 shows the results given by the system from secondaries arising from $\mathrm{K}^{+} \mathrm{p}$ interactions and gives examples of physics results. Our conclusions are presented in sect. 5 .

\section{Experimental configuration}

\subsection{Layout and use of the system}

The layout of the system is shown in fig. 1. The experiment uses the high energy rf separated hadron beam (S3) [10] to provide beams of positive kaons at a momentum of $70 \mathrm{GeV} / c$ and of negative kaons at a momentum of $110 \mathrm{GeV} / c$. At the higher momentum the separation is not complete; some pions and antiprotons also reach $\mathrm{BEBC}$. In order to provide a means of identifying the beam particles a tagging system [11] is installed in the last part of the beam line before BEBC. This system consists of two threshold gas Cherenkov counters for particle identification and two coded scintillator hodoscopes to determine the trajectories of the incident particles in the horizontal plane. Fast buffer storage allows up to sixteen successive particles to be recorded per fast spill of $3 \mu$ s with a time resolution of

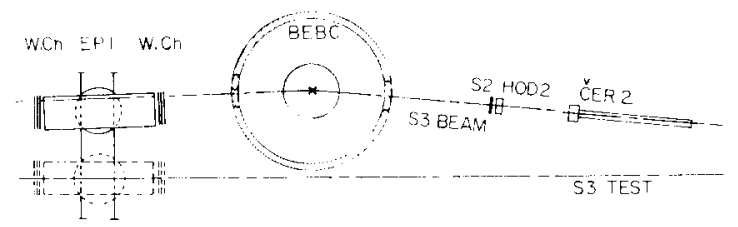

Fig. 1. Schematic layout of the BEBC hybrid system, showing the end of the $\mathrm{S} 3 \mathrm{rf}$ separated beam with the second hodoscope $\mathrm{H} 2$ and Cherenkov counter $\mathrm{C} 2$ of the tagging system, the $\mathrm{BEBC}$ bubble chamber and the EPI with its associated wire chambers. The position of the $\mathrm{S} 3$ test beam used for the calibration run is also shown. (Not to scale.)
$15 \mathrm{~ns}$. The hodoscope information is employed to predict the entrance point in the bubble chamber of each tagged particle.

The bubble chamber $\mathrm{BEBC}$ is a large conventional bubble chamber of $3.7 \mathrm{~m}$ diameter, which provides a $4 \pi$ acceptance for all types of events, good neutral particle detection efficiency due to its large size, and excellent event reconstruction quality due to the high magnetic field (35 kG) and good optical quality. A major limitation of such a chamber is the lack of charged particle identification for particles of momentum above about 1 $\mathrm{GeV} / c$. Below this momentum, protons can usually be separated from mesons by the departure of the trajectory from a helix due to energy loss, and there is some chance of identifying pions and kaons via their decays. To improve this situation the EPI was constructed to provide charged particle identification in the most interesting kinematic region $(20-100 \mathrm{GeV} / \mathrm{c})$. It is positioned downstream of the bubble chamber in the path of the fast secondary particles emerging through the exit windows. Wire chambers fixed to both ends of the EPI assist in tracking through the device and in momentum determination. Data from the tagging system, the E.PI and the wire chambers are collected by a dedicated computer and written onto magnetic tape for off-line analysis together with the measurements from the butble chamber pictures.

\subsection{External Particle Identifier}

The design of the EPI has been adapted to the given dimensions of the bubble chamber for optimum particle identification over a wide range of momenta. Construction details have been given elsewhere [1-4], and we will here summarize only those points that are of interest for the evaluation of the experimental results.

The device presents itself as an array of vertical ga: proportional counters stacked to a depth of 128 and a width of 32, forming a compact matrix of 4096 elements. The individual cells of $6 \times 6 \mathrm{~cm}^{2}$ section and $1 \mathrm{~m}$ height are formed by parallel stretched wires with $3 \mathrm{~cm}$ spacing and partitioned into 64 double modules as shown in fig. 2 . The resulting detection area is well matched to the spread of the secondary particles leaving BEBC and the depth is sufficient (with a maximum of 128 samples per track) to ensure good separation efficiency in the relativistic rise region of the ionization. We will, in the following, refer to any line of cells across the matrix as a "layer", numbering them from 0 to 127 and to any line parallel to the long axis as a "slice" with numbers 0 to 31 .

Two meter long printed circuit boards attached to the top of each layer contain the electronics for each cell, consisting of amplifier, integrator, 8-bit ADC, intermediate buffer and shift register read-out. The shift registers are chained and connected to one 16-bit paral- 




Fig. 2. Cell structure and layout of the EPI modules that form the $128 \times 32$ matrix of active cells. The position of the insensitive beam hole is indicated.

lel data link, which provides the data transmission to the control room. To ensure sufficient charge collection in each cell, integration is over the whole of the short spill time $(\approx 3 \mu \mathrm{s})$ of the fast extracted beam, so that the tracks pertaining to a given bubble chamber picture are all contained in the same block of data from the EPI with no additional time differentiation.

The device is essentially two-dimensional in nature. In the horizontal plane the hit cells give a pictorial view in the $32 \times 128$ raster of the traversing tracks, whereas no information as to where particles pass vertically is obtain from the cell matrix. Two tracks passing above one another will be seen as one track, resulting in unusable ionization values, which are eliminated in the data analysis. The secondary tracks are usually well separated at the position of the EPI by the diverging effect of the BEBC magnetic field, but problems occur with crossing background tracks. To prevent non-interacting beam tracks from obscuring the matrix, an insensitive area made of $4 \mathrm{~cm}$ long glass tubes cemented half-way up the signal wires in the first six active cells of each layer is located as shown in fig. 2. By steering the beam into this area, any fast forward secondaries passing above or below can be seen unambiguously. This "beam hole" is located at one side of the EPI, as the device is optimized to detect secondaries with the same charge as the incident particles. Such secondaries tend to be on one side of the beam tracks due to the BEBC field. It was found to be necessary, however, to have a fringe of the beam pass through the sensitive region to allow continuous monitoring of the beam particle ionization.
The complete device including the electronics is mounted on a chariot which permits movement into the optimum position for each beam momentum. The EPI can be moved vertically by $\pm 10 \mathrm{~cm}$ with respect to the beam plane, it can be rotated by $\pm 7^{\circ}$, and the whole assembly can be moved sideways as far as the test branch of the S3 beam (fig. 1). Due to hydrogen security all movements are made by compressed air motors under local control. The total weight of the assembly is $\sim 52 \mathrm{t}$, of which the detector modules contribute $16 \mathrm{t}$. Reproducibility of positioning, allowing for mechanical deformations, is to better than $1 \mathrm{~mm}$.

\subsection{External Particle Identifier wire chambers}

The two wire chambers fixed to the ends of the EPI are used to define accurately the entry and exit points of tracks traversing the EPI. They each consist of three planes, at $0^{\circ}$ (vertical wires) and $\pm 30^{\circ}$, with a surface of $2 \times 1 \mathrm{~m}^{2}$; fig. 3 gives technical details of the design. The wire spacing is $4 \mathrm{~mm}$ and the gas used is $70 \%$ $\mathrm{Ar}+30 \% \mathrm{CO}_{2}$. Each of the 3008 signal wires is equipped with a buffering capacity of 16 hits to allow the data to be partitioned into sixteen time slots with equal width of $0.35 \mu \mathrm{s}$ each for easier correlation of hits from the same fast spill. Fast random access memory is used for this storage. At the end of the spill a hardware scanner searches out the hits and compresses the information into words containing hit wire address and time slot number for subsequent transfer to the computer. In addition, within a time slot a fast OR signal from all wires of a traversed plane is provided for a cursory search. 

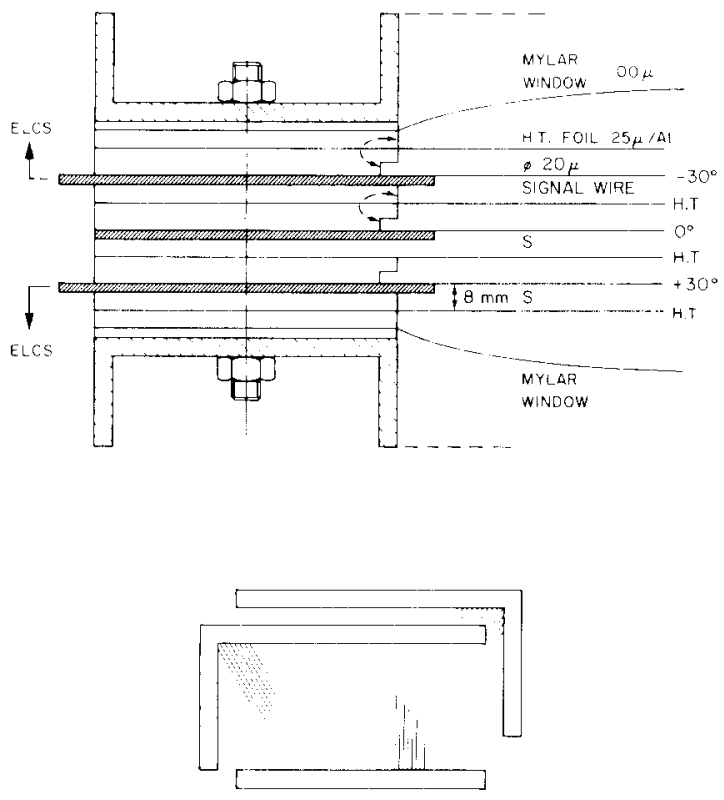

Fig. 3. Construction detail of an EPI wire chamber showing at the top the Vetronite/printed circuit sandwich structure of the frame and the three signal wire planes. For safety reasons (hydrogen security zone) the chambers are enclosed by thin $\mathrm{Al}$ sheets (dashed lines) fixed to the U-profiles and the $\mathrm{Ar} / \mathrm{CO}_{2}$ gas mixture of the chambers is exhausted into the spaces between these seals and the mylar windows.

\subsection{On-line control system}

Data acquisition is undertaken with a dedicated NORD10 computer, equipped with cartridge disc and a 1600 bpi magnetic tape unit running at a speed of 90 ips. Experimental input/output is generally through CAMAC, with direct memory access being employed where substantial quantities of data make this appropriate. Memory space of $64 \mathrm{~K} 16$-bit words is available and interactive work is possible via three terminals, one having graphic display capabilities.

The SINTRAN III real-time multi-tasking monitor is used to govern the data flow from the various sources through independent real-time tasks corresponding with each other for resource sharing via semaphores. The time structure of the extractions, consisting of two short spills two seconds apart every ten seconds, combined with the restricted and partly fixed lengths of the data input strings (e.g. 4096 bytes for the EPI and a maximum of $1 \mathrm{~K}$ bytes for the wire chamber per spill), made it superfluous to employ a complex memory manager for the data acquisition. Instead, fixed memory spaces are allocated to each of the data streams and the transfer of the data to tape is kept sufficiently fast to ensure that all tape writing is completed and the corresponding memory spaces are liberated before the subsequent spill.

The three major data acquisition tasks running in this way are connected to the Cherenkov tagging system, to the EPI and to the wire chambers. Since each task runs autonomously, the time, date and bubble chamber databox information are added to provide the necessary synchronization of the data streams in later off-line analysis. In the design of the hardware, emphasis was put on keeping the interfaces as similar as possible, so that these three tasks could also be kept almost identical in spite of the very different aspects of each of the devices. This has proved to be a great advantage for the software development effort. For the writing onto tape, independence of the data streams has been maintained, in that a separate logical record (decoupled from the standard 1020 word physical record length) is transferred by each device-task after the spill.

In addition to the data acquisition certain monitoring and control procedures were installed to ensure efficient running of the EPI. The flexible mylar entry



Fig. 4. On-line displays of an event: a) hit wires in the front wire chamber defining the entry points of particles into the detector; b) top view of the EPI with each dot representing a cell giving a measurement of ionization; c) as for a) for the back wire chamber defining the exit points of tracks from the EPI. 
and exit windows transmit the variations in air pressure to the gas inside the counters and induce corresponding changes in the gas amplification of the ionization signals. These changes are monitored with the aid of radioactive sources illuminating corner cells of the EPI, available since each layer has, in fact, been constructed with 34 cells to ensure full field homogeneity for the inner 32. These corner cells are connected to their own pulse height analysers with associated memories, which can be read out by the computer when the preselected number of counts has been reached. For an X-ray spectrum of ${ }^{55} \mathrm{Fe}$ and a $\beta$-spectrum of ${ }^{90} \mathrm{Sr}$ the relevant parameters of the spectra, e.g. position, half-height points, etc. are determined after application of an algebraic smoothing procedure to iron out fluctuations and these values are printed out periodically together with the internal pressure. Using the $\beta$ spectrum as reference, an active gain control by computer was implemented, which acts on the EPI high tension and thereby on the gas amplification in such a way as to keep the position of the spectrum constant. With reading and subsequent adjustment approximately every fifteen minutes, it was possible to maintain this position eliminating the need for off-line correction for pressure variations.

The on-line facility offers a variety of features such as pictorial displays, accumulation of distributions and print-outs to be activated by request for instant checking as well as for optimizing the running conditions at the start-up and during the experiment. As an illustration fig. 4 gives a pictorial view of particles traversing the EPI system. These requests and display routines run practically decoupled from the data acquisition streams on a low level of priority and cannot perturb or retard the data taking.

\subsection{External Particle Identifier calibration run}

Before using the EPI for secondary particle identification two sets of calibrations must be undertaken, namely the measurement, in the relativistic rise region,

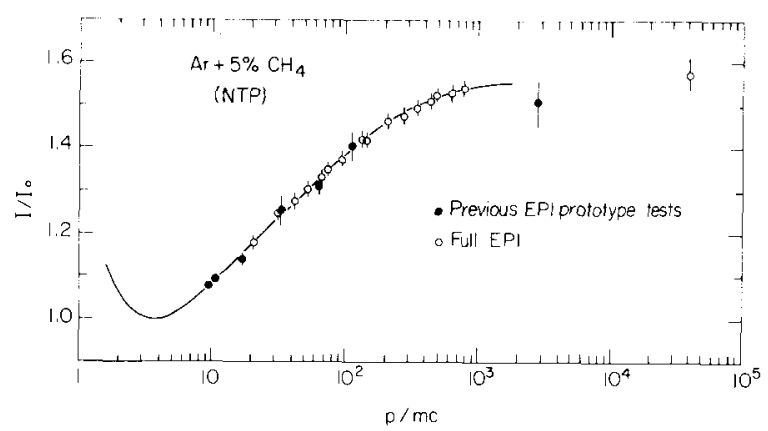

Fig. 5. Dependence of the ionization on the reduced momentum $p / m c$ in units of the ionization at minimum, as obtained in the test runs [5]. of the ionization deposited in the gas by a particle of known mass as a function of its momentum and also the individual response of each of the 4096 counters in the matrix to a given ionization deposited. The relativistic rise measurements, using a positive tagged pencil ray beam ( 33 upstream of $\mathrm{BEBC}$ ) of variable momentum passing centrally through slice 2 of the full EPI, have already been reported [5]. Fig. 5 gives the resulting dependence which forms the basis for the particle separation to be described in sects 3.6 and 4 . The measured points and their errors have been derived from the distributions of the mean of the lowest $40 \%$ of the ionization values (truncated mean) collected for each track. In this very low background beam, resolutions of $6 \%$ fwhm have been achieved *

The calibration of the individual cells was done by displacing the EPI laterally in $6 \mathrm{~cm}$ steps across the beam and concurrently measuring the 128 cells of the aligned slice in each position. A negative well-collimated $(3 \mathrm{~cm}$ width) pion beam of $50 \mathrm{GeV} / c$ momentum, the S3 test branch by-passing BEBC (fig. 1), was employed. The beam was centred on a slice with the aid of on-line displays of the top view of the EPI, since the associated wire chambers were not available for this run. The correct functioning of the apparatus was continuously monitored on-line; fig. 6 gives examples of an energy loss $(\mathrm{d} E / \mathrm{d} x)$ distribution and (truncated) mean energy loss over the whole slice for a given position. At an intensity of $\leqslant 1$ particle per spill ensuring a maximum of single particle signals, the needed statistics of $\sim 1000$ clean tracks was obtained in $\sim 6-8 \mathrm{~h}$ of running for each slice. Therefore, the use of the automatic gain control to counterbalance the pressure variations was of great importance. Fig. 7 shows the atmospheric pressure curve and the applied variation of the high tension, which kept the position of the ${ }^{90} \mathrm{Sr} \beta$ spectrum constant to within $\pm 0.5 \%$. Nevertheless, periodical cross checks with the beam centred on slice 11 showed a residual slow gain reduction for beam particles of the order of $0.4 \%$ per day. The reasons for this slow drift are not well understood, but the effect of this drift can be eliminated in the subsequent analysis, if the beam ionization has been periodically monitored.

As a measure of the individual cell responses, the positions of the most probable values of ionization in the $\mathrm{d} E / \mathrm{d} x$ distributions for each individual cell were taken. Selected results are shown in fig. 8. Equalization of electronics gain had been performed prior to mount-

* In general, we will quote resolutions as the (fractional) measured full widths at half-maximum, denoted by $W$, of truncated mean distributions. In our conditions these distributions are (in contrast to the ionization values themselves that follow the typical shape of the Landau distribution) nearly Gaussian $[1,2]$, where $W=2.36 \sigma$ and $\sigma$ is the standard deviation. 



Fig. 6. Typical on-line displays of the operation of a single slice during the calibration run (50 GeV/c $\pi^{-}$): (a) Distribution of the single cell ionization values as obtained from all cells of the slice. The horizontal scale is in ADC units divided by four. (b) Distribution of the means of the smallest $40 \%$ of ionization values in the slice. The horizontal scale is in ADC units. The main narrow peak corresponds to single particles, the auxiliary peak to two simultaneous particles passing through the slice. Note that distribution (a) includes these simultaneous particles.

ing the cards. The remaining general decrease of response toward the sides and toward the back of the device is predominantly caused by the effects of the EPI box on the overall electrostatic field distribution. Once these cell-to-cell differences are known, they have no effect on the final efficiency of the detector since they can be fully corrected for in the analysis of the particle data by renormalizing the measured ionization values from any cell to the overall average cell response.

Using the full data from slices 6 to 31 obtained over

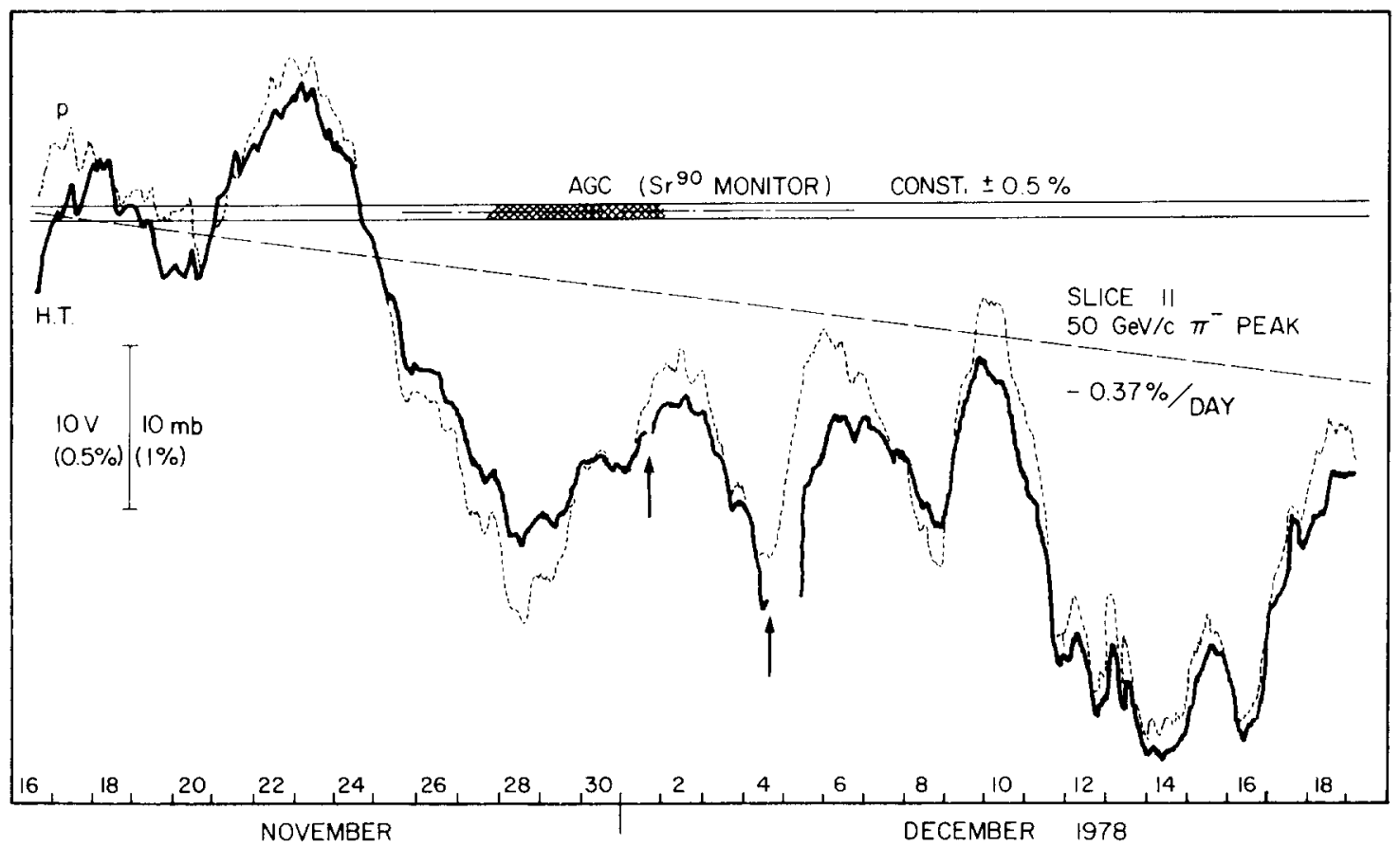

Fig. 7. Automatic Gain Control in the EPI during the calibration run. The scales for the barometric pressure $p$ (dotted line) and the high tension ht (full line) are shown on the left. The ht follows the atmospheric pressure variations keeping the position of the ${ }^{90} \mathrm{Sr}$ spectrum constant within $\pm 0.5 \%$. The remaining slow drift of the EPI response to traversing $50 \mathrm{GeV} / \mathrm{c} \pi^{-}$, as measured periodically in slice 11 , is indicated by the dashed line. The two arrows denote discontinuities due to power failures. 


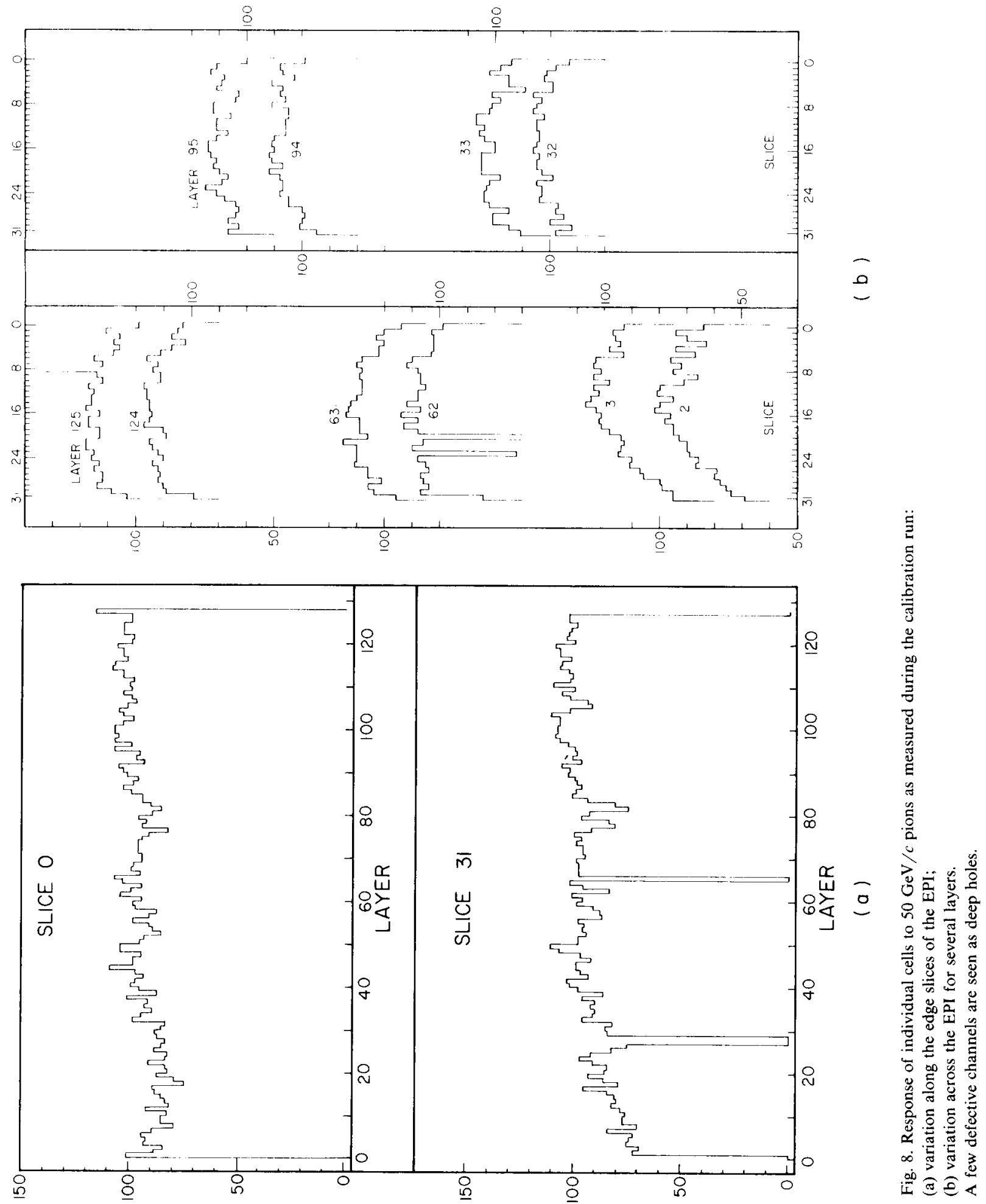




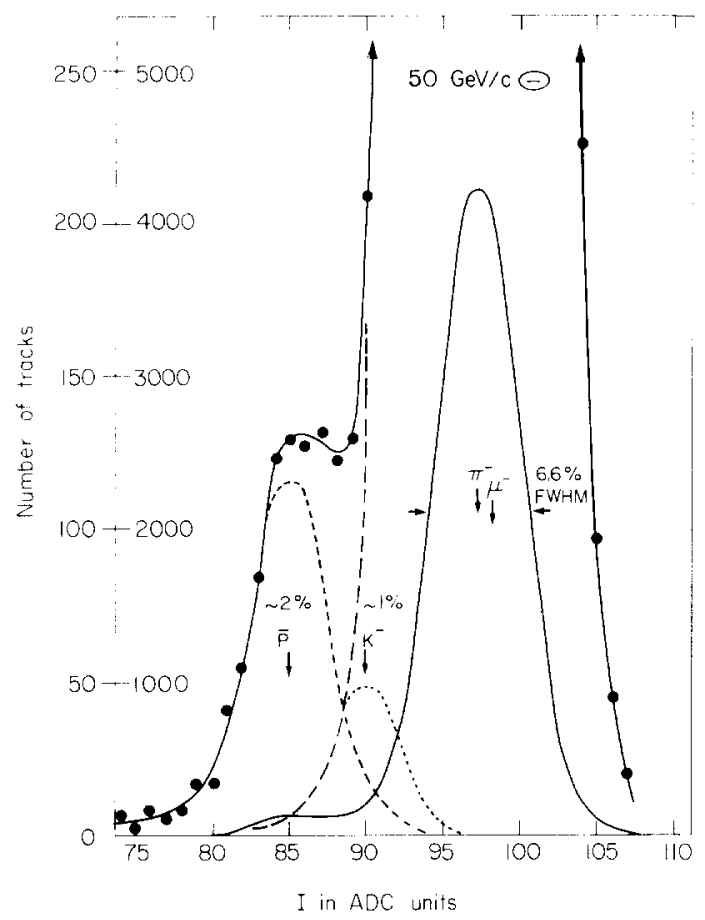

Fig. 9. Resolution of the EPI as obtained during the calibration run, using -31000 negative tracks of $50 \mathrm{GeV} / \mathrm{c}$. The thin line (right vertical scale) gives the full distribution with its $6.6 \%$ width. The lower part of this curve, magnified by a factor of 20 , is shown together with the measured points. Dashed lines indicate the deduced contribution of $\overline{\mathrm{p}}$ and $\mathrm{K}^{-}$.

ten days' running, the long-term resolution limits throughout the EPI were investigated. Plotted in fig. 9 is the truncated mean distribution (lowest $40 \%$ ) from over 31000 traversing beam tracks of fixed momentum. Cell by cell renormalization and long-term drift corrections are applied; double tracks and events with high local background have been excluded. The enlargement shows the bump caused by the estimated $2 \% \overline{\mathrm{p}}$ and $1 \% \mathrm{~K}^{-}$ contamination. The resulting overall ionization measurement resolution of the full device is $6.6 \% \mathrm{fwhm}$ for $50 \mathrm{GeV} / \mathrm{C}^{-\cdots}$; this is not corrected for a broadening due to a small $\mu^{-}$contamination.

The behaviour of the cell 'walls' (i.e. one wire every $3 \mathrm{~cm}$ ) was investigated by grazing the boundary between two slices with the beam as well as by angling the EPI at $3.5^{\circ}$ and $6.5^{\circ}$. No loss of ionization in the boundary region or leakage of ionization deposited in one slice into the neighbouring slice were recorded. It could also be shown that for particles crossing a slice boundary and partially traversing each of two adjacent cells of a given layer at this point, addition of the ionization deposited in both to form one full sampling for this layer did not alter the resulting distributions.

\subsection{Hadron experiments}

Two physics runs with the full BEBC hybrid system were undertaken, the first in November-December 1977 with a $110 \mathrm{GeV} / \mathrm{c}$ incident $\mathrm{K}^{-}$beam, the second in March-April 1979 which commenced with a continuation of the $110 \mathrm{GeV} / c$ exposure and was followed up by a $70 \mathrm{GeV} / c \mathrm{~K}^{+}$run (table 1 ). In this report we restrict the discussion to the results of the 1979 run, since it was statistically much more significant and included some improvements over 1977. Furthermore, for the results discussed in sect. 4 only the $\mathrm{K}^{+} \mathrm{p}$ data is presented, since this represents about four times the statistics of the $\mathrm{K}^{-} \mathrm{p}$ data.

For the $110 \mathrm{GeV} / \mathrm{c} \mathrm{K}^{-}$experiment the position of the EPI was chosen to have the beam centred $\sim 11 \mathrm{~cm}$ below the beam hole to ensure good monitoring conditions for beam particles and to survey the long-term drift. In the $\mathrm{K}^{+}$exposure it was preferred to keep the beam hole at the height of the beam, but to displace the EPI horizontally so that part of the beam traversed the slices adjacent to the beam hole. Thus it was possible to

Table 1

Summary of the experimental runs.

\begin{tabular}{|c|c|c|c|c|c|}
\hline Date & Beam & Particles & $\begin{array}{l}\text { Energy } \\
(\mathrm{GeV} / c)\end{array}$ & Purpose & Remarks \\
\hline $\begin{array}{l}\text { Nov.-Dec. } \\
1977\end{array}$ & $\mathrm{~S} 3$ beam & $\mathrm{K}^{-}$ & 110 & $\mathbf{K}^{-}$p physics & $\begin{array}{l}\text { The EPI wire chambers were not available. } \\
\text { More rudimentary information from auxiliary } \\
\text { wire chambers used instead. No automatic } \\
\text { gain control for gas amplification }\end{array}$ \\
\hline $\begin{array}{l}\text { Nov.-Dec. } \\
1978\end{array}$ & $\begin{array}{l}\text { S3 test } \\
\text { branch }\end{array}$ & $\begin{array}{l}\text { Unsepar. } \\
\pi^{-}\end{array}$ & 50 & $\begin{array}{l}\text { calibration of individ. } \\
\text { cell response }\end{array}$ & \\
\hline March 1979 & $\mathrm{~S} 3$ beam & $\mathrm{K}^{-}$ & 110 & $\mathrm{~K}^{-} \mathrm{p}$ physics & full operating system \\
\hline April 1979 & S3 beam & $\mathrm{K}^{+}$ & 70 & $\mathbf{K}^{+}$p physics & full operating system \\
\hline
\end{tabular}


determine and monitor the (truncated) mean ionization loss of the beam particles passing through slices 3 or 4 at $110 \mathrm{GeV} / c$ and 6,7 or 8 at $70 \mathrm{GeV} / c$ using only rudimentary local cleaning. Since the momentum is known, the corresponding value for minimum ionizating particles $I_{0}$, needed as the reference value for mass identification, follows from fig. 5 .

Analysis of the full data of both runs gave, for the long-term drift of the $I_{0}$ measurement, values of $-0.5 \%$ per day in the case of the $110 \mathrm{GeV} / c$ experiment and $-0.08 \%$ per day for the $70 \mathrm{GeV} / c$ run. The decrease in drift is linked to the sequence of experiments, the 70 $\mathrm{GeV} / c$ run having occurred in more stabilized conditions.

\section{Off-line analysis}

\subsection{Introduction}

There are three main problems to be solved in order to use the EPI data in conjunction with measured BEBC events. The first is to follow the secondary particles that leave $B E B C$ through a non-uniform magnetic field and a series of media of differing densities to the position of the EPI, and there to associate the predicted points of entry and exit with the corresponding hits in the EPI wire chambers. The second is to find tracks in the EPI by a process of pattern recognition and to associate these tracks to those extrapolated from BEBC. The third problem is to obtain a clean (i.e. uncontaminated by crossing tracks) set of ionization values for those tracks which have been found in the EPI and associated to tracks leaving $\mathrm{BEBC}$, to obtain a measurement of the ionization of these tracks for subsequent use in mass identification. In addition, the wire chambers together with the BEBC magnetic field are used as a spectrometer to obtain an improved measurement of the track momenta where appropriate.

\subsection{Program logic and data flow}

The event input consists of the point fit, track fit and mass fit results from the measurements of an interaction in BEBC processed with the standard CERN HYDRA geometry program [12]. These data are searched for candidate tracks for following to the EPI (i.e. all those tracks that leave the chamber without interacting). If any such tracks are found they are followed through the magnetic field to the position of the EPI and tested to see if they enter the device.

If any tracks do enter the EPI, the EPI wire chamber data is read in. The predicted hits are compared with the reconstructed points in the wire chambers, and if they correspond, the hits are associated.

If any track associates to the EPI, the EPI ionization data is read in and the pattern recognition performed. If this is successful, the resulting ionization data for the track(s) is analyzed. Finally, a printed summary is produced, and the processed event including the pertinent information from the EPI and the wire chambers is written onto the output tape.

\subsection{Extrapolation to EPI and association with wire cham- bers}

Each candidate track is followed through the magnetic field outside the chamber towards the EPI, taking into account multiple scattering and energy loss in the various materials the particle traverses. If, according to the extrapolation, the candidate track does not pass through the first wire chamber of the EPI, it is abandoned. Where it intersects any wire chamber plane, the point of intersection is computed ready for association with the wire chamber hits.

The most important factor governing the accuracy of the tracking is the accuracy of the magnetic field map in the region outside $B E B C$, in particular the vertical component of the field. The critical region is in the hole in the BEBC shielding, where the field is highly irregular and the vertical component reaches $-5 \mathrm{kG}$ in the opposite sense to that of the field in the chamber. The residual uncertainties in the field map reduce the efficiency of association of extrapolated tracks to the EPI: small changes in the assumed magnitude of the field in the hole region sometimes shift a track by one slice in the EPI, thereby complicating the analysis.

The position in space of the EPI and its wire chambers is given by a set of external fiducial marks fixed to the outside of the box at known points with respect to internal marks. During a run, when the EPI has been positioned in its final running position, the external fiducials are surveyed, enabling the position of the actual EPI cell matrix to be determined. The position of the wire planes of the EPI wire chambers are located in a similar fashion.

Using this survey information, the predicted positions of the tracks passing through the wire chambers (from extrapolation from BEBC) can be associated with the reconstructed points in the wire chambers. The software used for the extrapolation and association is based on that described in ref. 13. Small systematic errors in the tracking due to uncertainties in the magnetic field map and in the results of the survey were corrected by using comparisons between the positions of tracks as predicted by the tracking and as measured in the wire chambers.

After these corrections, using a large sample of tracks, the momentum measurement as obtained in BEBC can be improved by using the spectrometer-like information provided by the wire chambers and the BEBC magnetic field. The results of the momentum determination are illustrated in fig. 10. 


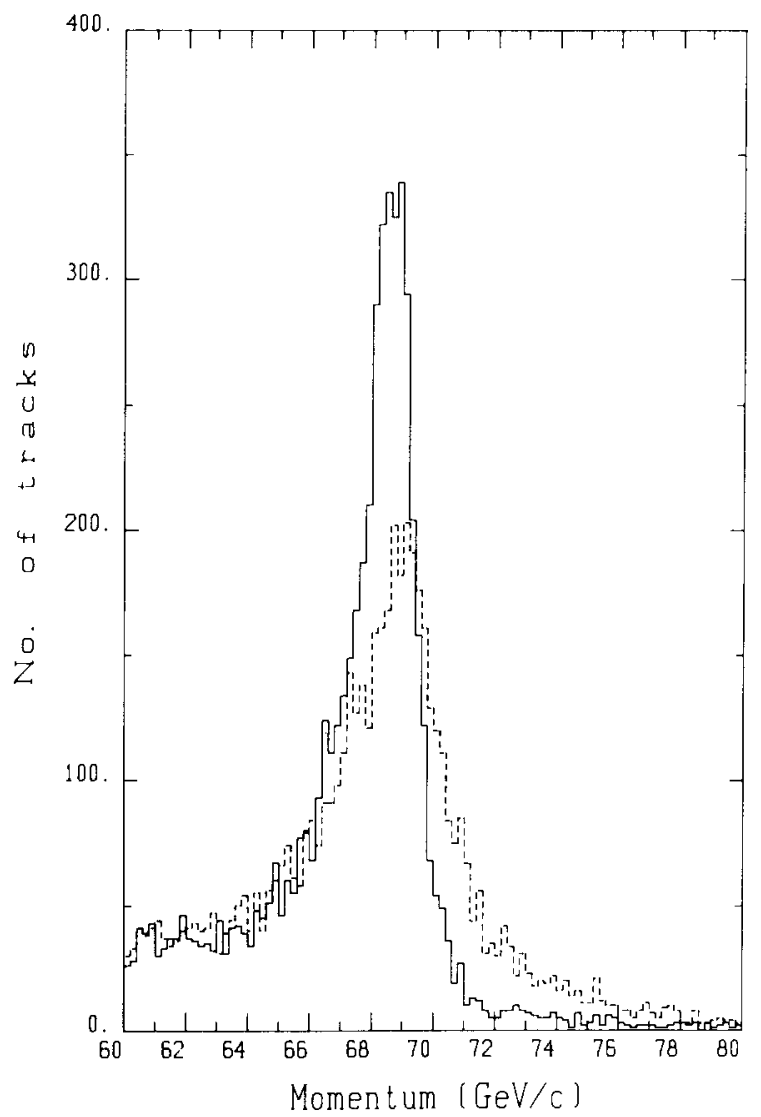

Fig. 10. Distribution of momentum of high energy positive secondary tracks from $\mathrm{K}^{+} \mathrm{p}$ interactions at $70 \mathrm{GeV} / c$ in BEBC: dashed histogram from $B E B C$ measurements alone; solid histogram after including measurements from the EPI wire chambers.

\subsection{Pattern recognition and association of tracks in EPI}

This part of the program finds tracks in the EPI cell matrix without using any external information. In particular, the background tracks which do not cross the wire chambers can only be found in this way. The problem is two-dimensional in nature, and for the purposes of the pattern recognition the EPI is considered as a matrix of points in a plane where the unit of length is a cell length $(6 \mathrm{~cm}$ in real space), resulting in a rectangular array 32 units by 128 units. As the stray magnetic field from $B E B C$ is only $\sim 100 \mathrm{G}$ in the region of the EPI it is sufficient to approximate all tracks by straight lines for pattern recognition purposes.

The EPI data is scanned slice by slice to find "strings" of hit cells, a string being at least three consecutive cells with non-zero ionization values. When the complete array has been scanned and all the strings found, the program links strings together to form tracks. Different algorithms are applied for short ( 3 to 10 cells), medium ( 10 to 40 cells) and long ( $>40$ cells) strings. In the case of short and medium strings it looks for any string of more than three cells length as a possible starting point for a track and then checks in the slices adjacent to the start string for strings possibly belonging to the same track. If such strings are found, a linear equation can be constructed using the points of cross-over between slices as points on the line of the track. As these points can be uncertain (the strings can overlap by one cell, not overlap, or there could even be a missing cell) a tolerance is used when calculating the location of more strings until sufficient cross-over points (usually four are enough) have been found and the track direction calculated more precisely. When the track equation is established it is followed as long as strings are found which fit the equation.

In the case of a medium length string for which no track can be found by the above method, or for a long initial string, a different procedure is applied. The program searches for a single transition between slices and calculates a "road" within which the track could lie. As there are very few transitions with tracks of this type, this procedure is sometimes not very successful. The same is true for tracks passing completely through one slice, for which no transitions exist and the road is equal to the slice itself with no further information on the position within the slice. For these tracks if often proves better to find them using the predictions from the tracking.

When the array has been completely scanned and a set of "EPI tracks" has been established, the program attempts to associate these tracks with the tracks from $B E B C$ that associated with reconstructed points in the wire chambers (note, in many cases the found EPI track will be a background track, so no association is expected). Also at this stage the program searches for strings that lie on the predicted track, but that may not have been linked together into an EPI track in the independent pattern recognition process. In some cases this tracking prediction method duplicates the results of the pattern recognition process. In other cases, for example the types of tracks difficult for the pattern recognition as discussed above, this method finds new tracks in the EPI.

\subsection{Removal of ionization samples biased by crossing tracks}

Crossing tracks increase the ionization values in those cells of the desired track that they traverse. The ionization values from these doubly hit cells must be excluded from subsequent analysis. Two cleaning procedures are applied. The first removes the cells that are common to the current track string and any other track found in the 
(o)



(b)



$\bigotimes=$ eliminated cells

$\square=$ cells with background hits

Fig. 11. Examples to show the basic configuration of background hits that are the basis of the local cleaning algorithms: (a) with three adjacent cells on one side of a track and (b) with one on side and two on the other side of a track.

pattern recognition. The second procedure is purely local to the track string considered and is designed to remove crossing background that has not been picked up in the pattern recognition. The principle of this "local cleaning" is seen in fig. 11, which shows the types of sequences of the hit background cells that cause the removal of a cell in the track string of interest. Fig. 12 shows an example of an event with a cleaned associated track after the application of all the above procedures.

\subsection{Ionization analysis}

The retained ionization values for a given track are corrected for the individual cell response as measured during the calibration run and for the long-term drift. A minor correction is applied for the true path length of the particle in a cell. In most cases this factor does not exceed 1.05 , as the tracks generally have small azimuthal angles and nearly zero dip. A dependence on the track orientation was found empirically by the analysis of the ionization measured on a sample of tracks in selected angular intervals. A correction factor of the form $\alpha\left(n_{\mathrm{s}}\right.$ $-1) /\left(x_{2}-x_{1}\right)$, where $n_{\mathrm{s}}$ is the number of strings, $x_{1}$ and $x_{2}$ are respectively the entry and exit coordinates of the track along the longitudinal axis of the EPI and $\alpha$ is an empirical constant, was applied.

If the proportion of maximum ionization values in a string exceeds a fixed limit, it is assumed to have been traversed by two particles and this string is excluded. A similar cut rejects whole tracks caused by two particles.

A minimum requirement of at least 50 remaining cells per track is imposed for mass identification, as the resolution becomes too poor below this number of samples. The (truncated) mean, $I$, of the smallest $40 \%$ of the retained ionization values is calculated and related to the truncated mean, $I_{0}$, for minimum ionizing par-

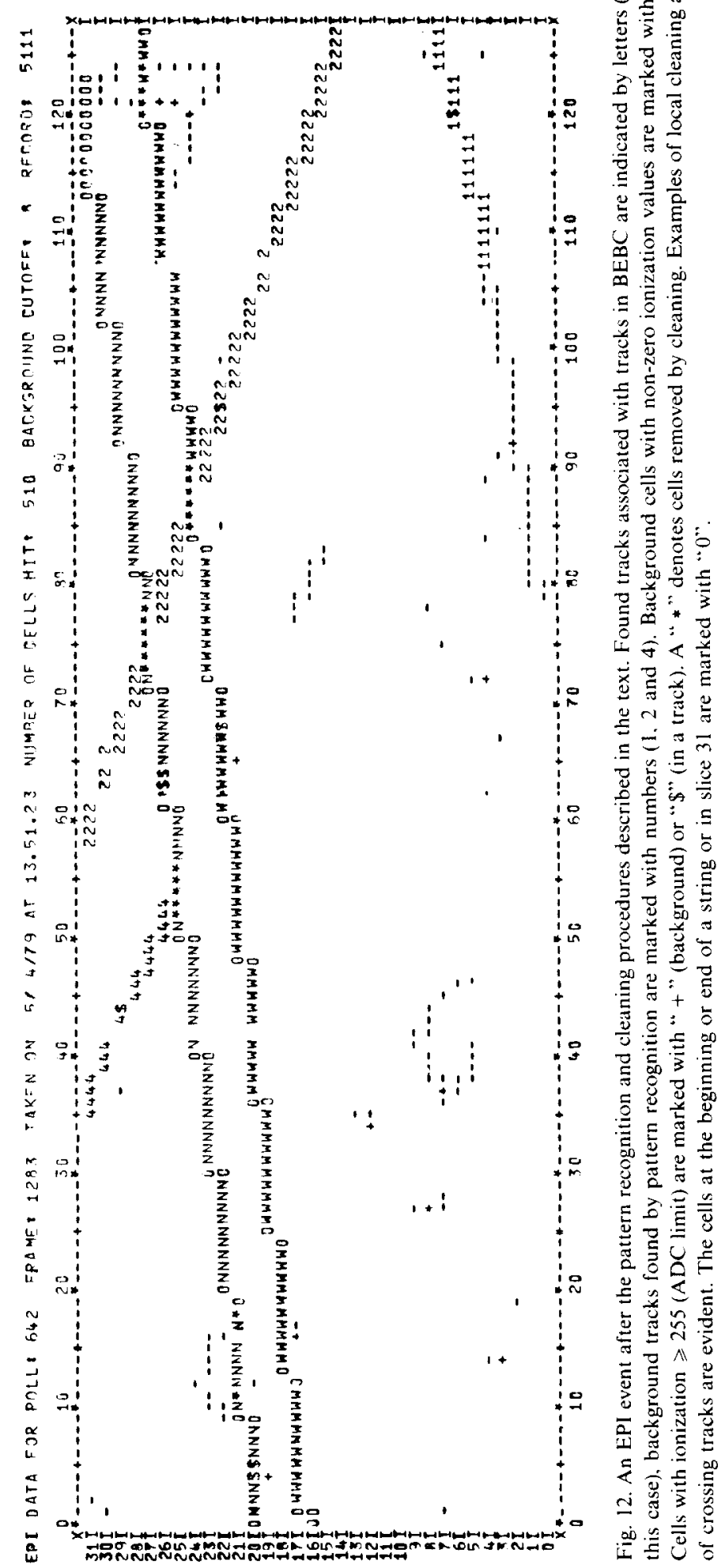


ticles, as derived from beam tracks in the manner discussed, to produce the measured value of $I / I_{0}$.

Since the ionization distributions obtained in this way are approximately Gaussian, it is possible to compute a $\chi^{2}$ for each mass hypothesis ( $i=$ pion, kaon or proton) with

$\chi_{i}^{2}=\frac{\left[\left(I / I_{0}\right)^{\mathrm{M}}-\left(I / I_{0}\right)_{i}^{\mathrm{T}}\right]^{2}}{\Delta^{2}}$,

and the corresponding one degree of freedom $\chi^{2}$ probability, $P_{i}^{\mathrm{EPI}}$. Here $\left(I / I_{0}\right)^{\mathrm{M}}$ is the measured value of ionization, $\left(I / I_{0}\right)_{i}^{\mathrm{T}}$ is the predicted most probable value for this mass $\left(m_{i}\right)$ and momentum $(p)$. The value of $\left(I / I_{0}\right)_{i}^{\mathrm{T}}$ is obtained from the relativistic rise curve shown in fig. 5 , parametrized by three polynomials of the form

$$
\left(I / I_{0}\right)_{i}^{\mathrm{T}}=a+b \log _{\mathrm{e}}\left(p / m_{i}\right)+c\left[\log _{\mathrm{e}}\left(p / m_{i}\right)\right]^{2} \text {. }
$$

The three regions of $\log _{\mathrm{e}}(p / m)$ and the corresponding coefficients $a, b, c$ are shown in table 2 . from

The Gaussian-like error, $\Delta$, in eq. (1) is estimated

$\Delta^{2}=\left[\frac{d\left(I / I_{0}\right)_{i}^{\mathrm{T}}}{d p} \sigma_{p}\right]^{2}+\left[\frac{1}{2.16} W_{N}\left(\frac{I}{I_{0}}\right)^{\mathrm{M}}\right]^{2}$.

The first term in equation (3) represents the uncertainty in $\left(I / I_{o}\right)_{i}^{\mathrm{T}}$ due to the track momentum error, $\sigma_{p}$.

The second term in eq. (3) is the expected standard deviation corresponding to the measurement $\left(I / I_{0}\right)^{\mathrm{M}}$ from a sample of $N$ clean cells. We assume that the fractional width $W_{N}$ does not vary with mass or momentum over the range of interest for a given $N$, as found earlier [5]. The $\mathrm{N}$ dependence of $W$ is parametrized as the sum of two terms

$W_{N}=W_{128}\left(\frac{N}{128}\right)^{-0.45}+w$.

The first term describes the statistical effect of a reduced number of ionization samplings. It was obtained from measurements of $20 \mathrm{GeV} / c \pi^{+}$passing through a slice of the EPI, giving the results shown in fig. 13. The value $W_{128}=6.6 \%$ as found in the calibration was used throughout. The second term, $w$, is a small correction related to non-statistical effects such as difficulties in the removal of background, in the local cleaning etc. Its parametrization is discussed in sect. 4.7.

Table 2

Coefficients of the empirical equation (2).

\begin{tabular}{llll}
\hline Range of $\log _{\mathrm{e}}(p / m)$ & $a$ & $b$ & $c$ \\
\hline$<2.25$ & +1.096 & -0.170 & +0.072 \\
$2.25-4.44$ & +0.794 & +0.125 & +0.001 \\
$>4.44$ & +0.432 & +0.298 & -0.020 \\
\hline
\end{tabular}



Fig. 13. Dependence of the fractional width on the number of cells, as derived from data of the $20 \mathrm{GeV} / c$ test run [5]. The full curve decreases as $N^{-0.45}$.

\section{Experimental results}

\subsection{Introduction}

The results presented here are based on a sample of 52525 events from the $70 \mathrm{GeV} / c \mathrm{~K}^{+}$data (table 1). This sample contains 9283 "accepted EPI tracks" defined as positive tracks originating from the interaction, with $p>10 \mathrm{GeV} / c$, passing through the EPI and yielding at least 50 clean cells. In addition, there are 291 well defined tracks arising from the decay of $\mathrm{K}^{0}$ 's or $\Lambda$ 's identified in BEBC. Of the events with accepted EPI tracks, only $\sim 10 \%$ have more than one such track.

\subsection{External Particle Identifier acceptance}

In order to characterize the efficiency of the EPI in a given experiment, we define three ratios $R_{1}, R_{2}$ and $R_{3}$. They are obtained from the number $N_{0}$ of secondary tracks of the same charge as the beam with $p>10$ $\mathrm{GeV} / c$ from events seen in BEBC, the number $N_{\mathrm{F}}$ of tracks traversing the EPI for a length exceeding 50 cells, and the number $N_{\mathrm{A}}$ of accepted EPI tracks as follows: the overall acceptance $R_{1}=N_{\mathrm{A}} / N_{0}$, the geometrical acceptance $R_{2}=N_{\mathrm{E}} / N_{0}$ and the identification efficiency $R_{3}=N_{\mathrm{A}} / N_{\mathrm{E}}$.

These quantities are shown in fig. 14 as a function of laboratory momentum $p_{\text {lat }}$. The average overall acceptance in the region $25-65 \mathrm{GeV} / c$ is $35 \%$. The geometrical acceptance $\left(R_{2}\right)$ falls off rapidly below $25 \mathrm{GeV} / c$, but is roughly constant at $-65 \%$ above this region. The identification efficiency $R_{3}$ shows a decrease for momenta below $\sim 25 \mathrm{GeV} / c$ due to the shorter average distance traversed in the EPI, and a steady decrease at high momenta due to the higher density of tracks and the related difficulties in the pattern recognition and cleaning of high momentum tracks. It is important to note that the identification efficiency $R_{3}$ depends 


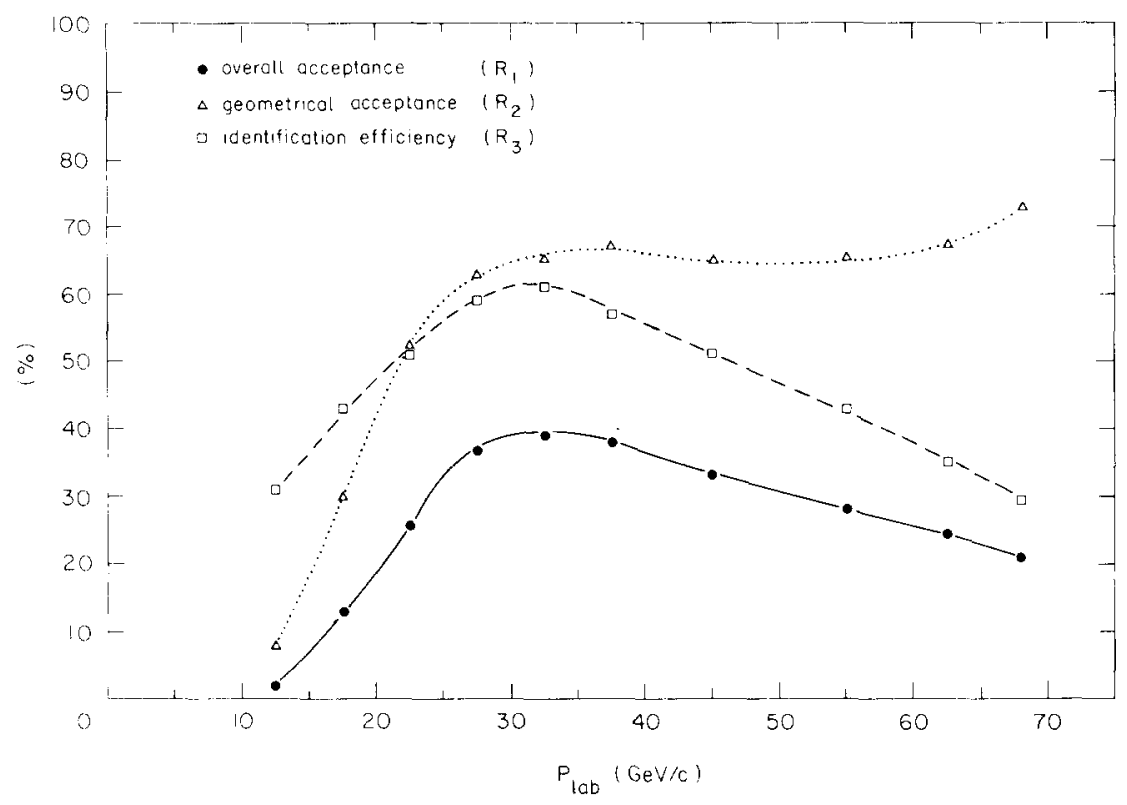

Fig. 14. The overall acceptance $\left(R_{1}\right)$, geometrical acceptance $\left(R_{2}\right)$ and identification efficiency $\left(R_{3}\right)$ as a function of laboratory momentum. The curves are to guide the eye.

strongly on the beam intensity, higher intensities causing serious background problems. For an average beam intensity (for BEBC pictures with measured events) of $\left\langle n_{\mathrm{b}}\right\rangle=2.5$ the plateau value of $R_{3}$ (at $-35 \mathrm{GeV} / c$ ) is $\sim 80 \%$, whereas for $\left\langle n_{\mathrm{b}}\right\rangle=11$ this value decreases to $-30 \%$. For the data presented, with $\left\langle n_{\mathrm{b}}\right\rangle=6.1$, the corresponding plateau value is $-60 \%$.

A similar investigation of the acceptance as a function of the transverse momentum $p_{\mathrm{t}}$ shows that the overall acceptance is essentially independent of $p_{\mathrm{t}}$. For the physics results presented here the distributions have been corrected by the overall acceptance weight, $1 / R_{1}$.

\subsection{Ionization results from specific samples}

A check of the EPI operation is afforded by the uniquely identified secondaries of $\mathrm{V}^{0}$ decays. Fig. 15a presents $I / I_{0}$ vs $p_{\text {lab }}$ for the positive particles arising from the decays of $\mathrm{K}^{0}$ 's and $\Lambda$ 's uniquely identified by kinematic fitting. They are seen to cluster around the corresponding predicted curves.

Another useful sample highly enriched in a given particle type is produced by a small deuteron contamination in the $\mathrm{K}^{+} 70 \mathrm{GeV} / c$ beam. In this experiment all events were measured and the tagging system

Fig. 15. The ionization measurements as a function of $p_{1 \mathrm{ab}}$ for positively charged secondaries from various samples. The curves are derived from fig. 5: (a) from decay tracks from identified $\mathrm{K}^{0}$ 's and $A$ 's $(N \geqslant 50)$; (b) from events highly enriched in the

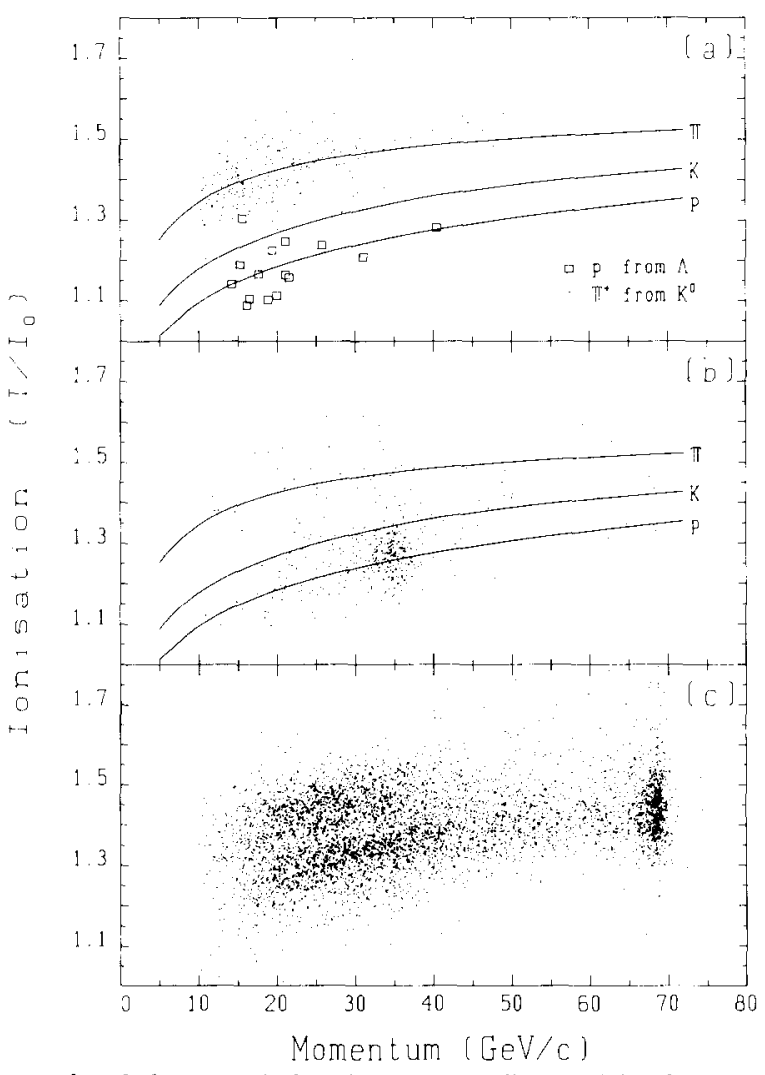

sample of deuteron induced events as discussed in the text $(N \geqslant 50)$; (c) from the total sample of $\mathrm{K}^{+}$induced events with at least 80 clean cells. 
was used a posteriori to identify those events $(-2.5 \%$ of the sample) that were deuteron induced. For the sample discussed above and in the following sections these deuteron induced events were removed, leaving a pure $\mathrm{K}^{+}$induced sample. For the sample discussed here, we select those events with the incident particle tagged by the Cherenkov system as having velocity lower than a kaon (i.e. mass greater than kaon). These events are roughly $\sim 50 \%$ deuteron induced and the rest are mainly $\mathrm{K}^{+}$induced events, but misidentified due to the inefficiencies of the tagging system. Any proton contamination in the beam is negligible. Many of the deuteron induced events yield a secondary proton of $-35 \mathrm{GeV} / \mathrm{c}$ (one half of the beam momentum), produced from the deuteron stripping reactions in which the neutron interacts with the target proton. The $I / I_{0}$ measurements of the secondary positive tracks in the EPI from these tagged events, presented in fig. 15b, shows a strong clustering of values around the proton curve at $\sim 35$ $\mathrm{GeV} / \mathrm{c}$. The background of points on this plot is due to secondaries arising from other $d p$ reactions and from the misidentified beam $\mathrm{K}^{+}$'s in this sample.

As another example of EPI results, fig. 15e shows the plot of $I / I_{0}$ vs $p_{\text {lah }}$ for all accepted EPI tracks with $N \geqslant 80$ clean cells (from the pure $\mathrm{K}^{+}$induced final sample). The bands of points corresponding to pions and kaons are clearly visible. At high momenta the predominance of kaons, due to elastic and diffractive reactions, is evident. This type of distribution, but with the sample of tracks with $N \geqslant 50$, forms the basis of the mass identification discussed in the following section.

\subsection{Mass identification}

The $\chi^{2}$ probabilities $P_{i}^{\text {EPI }}$ defined in sect. 3.6 could be used directly for identification if the total sample consisted of equal numbers of pions, kaons and protons. In the real situation, however, the relative populations of the three kinds of particles are very dissimilar and vary strongly with momentum. It is thus necessary to first determine the relative population fractions as a function of momentum (by one of the two methods described below). Then, we can make a mass identification on a track by track basis. Two approaches have been investigated, one based on a $\chi^{2}$ probability (confidence level) choice method and the other on a continuous probability distribution weighting method. In both methods we take into account the different relative populations of $\pi, \mathrm{K}$ and $\mathrm{p}$ as a function of momentum by using this population fraction as an a priori probability factor to be combined with the $\chi^{2}$ probability defined in sect. 3.6 (for the first method) or with the Gaussian distribution (for the second method).

\subsection{Determination of the relative fraction of particle types}

As already mentioned, the $I / I_{0}$ measurements from $\mathrm{N}$ clean cells follow closely a Gaussian distribution. The expected resolution functions generated as described in sect. 4.7 for the distribution of $N$ experimentally obtained in a given momentum interval also follow a roughly Gaussian shape. The $I / I_{0}$ distributions from seven samples in successive momentum intervals have therefore been fitted to the sum of three Gaussian distributions described by nine parameters, the peak positions $\left\langle I / I_{0}\right\rangle_{i}$, the standard deviations $\sigma_{i}$ and the numbers of tracks contributing $n_{i}$ (with the condition that $\sum n_{i}=n$, the total number of tracks) and where $i=\pi, \mathrm{K}, \mathrm{p}$.

The Gaussian fits have been performed on the samples with at least 50 clean cells and (for the resolution study) with at least 80 clean cells. Due to the limited statistics and the degree of overlap of the three Gaussians the fits do not readily converge in all momentum regions. To restrict the number of free parameters for the fits, the condition that the fractional widths are independent of particle type [5] was applied,

$\sigma_{\pi, \mathrm{p}} /\left(I / I_{0}\right)_{\pi \cdot \mathrm{p}}^{\mathrm{T}}=\sigma_{\mathrm{K}} /\left(I / I_{0}\right)_{\mathrm{K}}^{\mathrm{T}}$

(for each given momentum interval). The fitted values of the peak positions shown in fig. 16 are seen to be in good agreement with the relativistic rise curves. In a second stage, the peak positions were fixed to the predicted values $\left(I / I_{0}\right)^{\mathrm{T}}$ [eq. (2)]. Thus, only three free parameters, $\sigma_{\mathrm{K}}, \mathrm{n}_{\pi}$ and $n_{\mathrm{K}}$ remain in the fit. To remove the effect of the momentum dependence of $\left(I / I_{0}\right)_{i}^{\mathrm{T}}$ over the momentum interval selected, fits were made to the distribution of $\left[I / I_{0}-\left(I / I_{0}\right)_{\mathrm{K}}^{\mathrm{T}}\right]$, the second term being

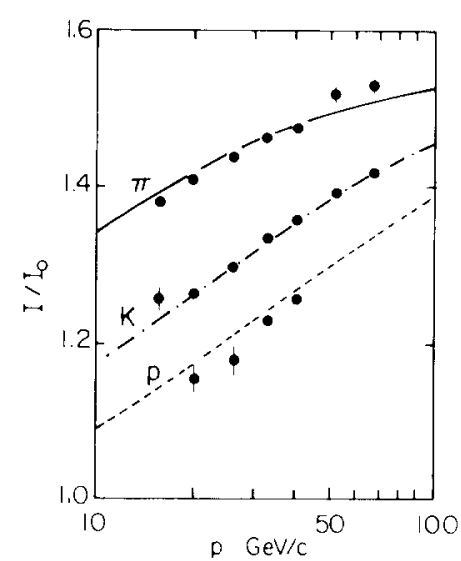

Fig. 16. Peak values of $\left\langle I / I_{0}\right\rangle$ for pions, kaons and protons obtained from Gaussian fits to the truncated mean distributions with at least 50 clean cells in seven successive momentum intervals. When not shown, the errors are of the size of the points. The curves are derived from fig. 5. 

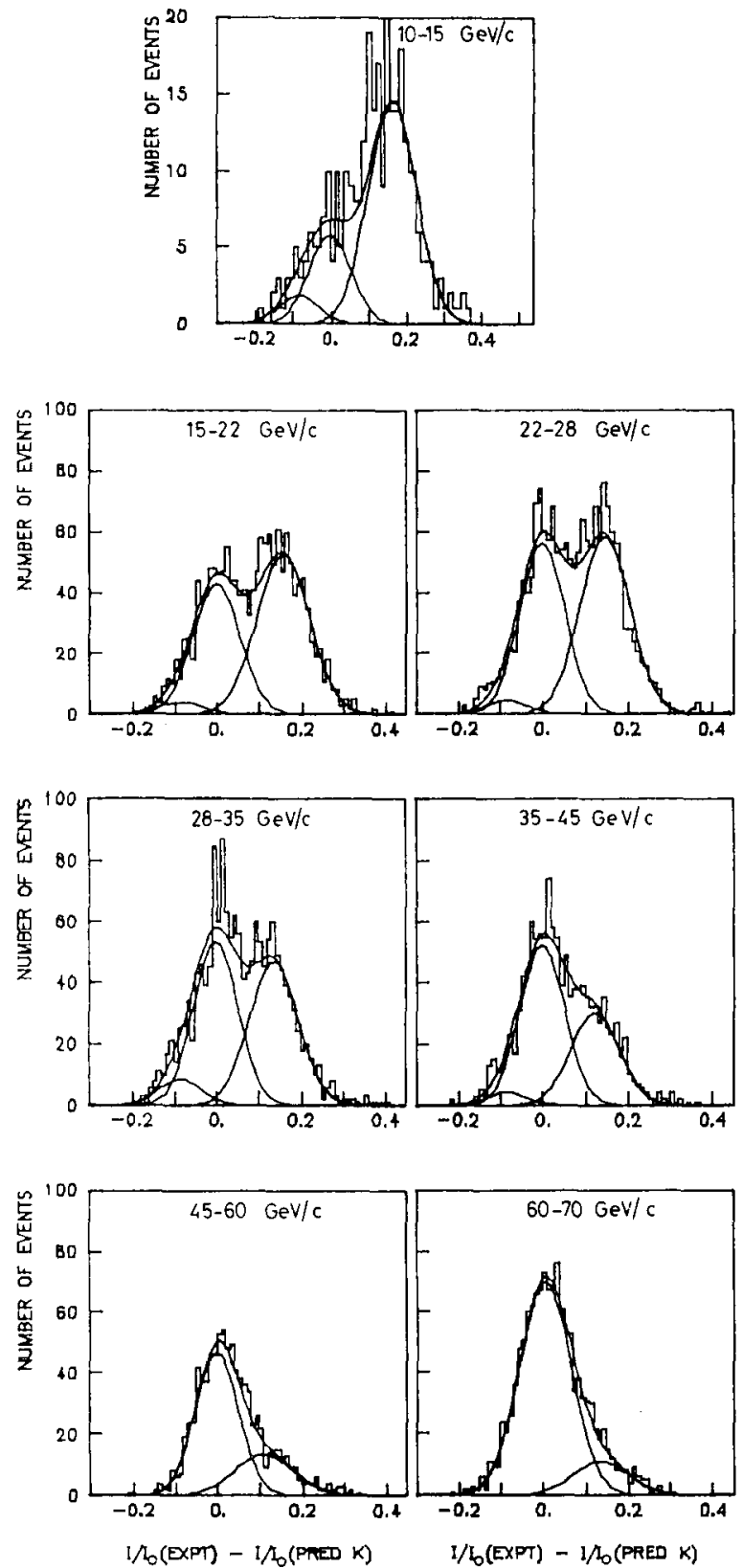

Fig. 17. The distributions of $I / I_{0}-\left(I / I_{0}\right)_{\mathrm{K}}^{\mathrm{T}}$ from tracks with at least 50 clean cells, in seven momentum intervals. The upper curve on each distribution is the result of the fits to the three (or two for $p_{\text {lab }}>45 \mathrm{GeV} / c$ ) Gaussians as described in the text. The lower curves show the contributions of $\pi^{+}, \mathrm{K}^{+}$and (for $p_{\text {lab }}>45 \mathrm{GeV} / c$ ) protons to the fits. The $\mathrm{K}$ curve is centred at zero, with $\pi$ 's to the right and protons to the left.

evaluated using the measured momentum of each track.

The results of these fits are shown by the curves in fig. 17 and the corresponding particle fractions $f_{i}=n_{i} / n$



Fig. 18. The fitted particle population fractions $f_{i}$ as a function of $p_{\text {lab }}$. The curves correspond to eq. (5).

are presented in fig. 18. For $p_{\text {lab }}>45 \mathrm{GeV} / c$ there is no evidence for protons in the $I / I_{0}$ distributions, so $n_{\mathrm{p}}$ was set to zero (leaving only two parameters). The particle fractions have been parametrized as

$f_{\pi}=\left(92.6-1.92 p_{\text {lab }}+0.0115 p_{\text {lab }}^{2}\right) \times 10^{-2}$,

$f_{\mathrm{K}}=\left(-4.92+2.22 p_{\text {lab }}-0.0135 p_{\text {lab }}^{2}\right) \times 10^{-2}$,

and

$f_{\mathrm{p}}=\left(12.2-0.304 p_{\text {lab }}+0.002 p_{\text {lab }}^{2}\right) \times 10^{-2}$,

where $p_{\text {lab }}$ is expressed in $\mathrm{GeV} / c$.

An alternative method to determine the values $f_{i}$, particularly suited to a situation with limited statistics, is to use an iterative procedure as follows:

1) An initial estimate for $n_{i}\left(n_{i}^{0}\right)$ is obtained by choosing for each track the highest probability $P_{i}^{\mathrm{EPI}}$. Then, compute $f_{i}^{1}=n_{i}^{0} / n$.

2) Estimate $n_{i}^{1}$ by choosing for each track the highest probability $P_{i}^{\mathrm{EPI}} f_{i}^{1}$. Then compute $f_{i}^{2}=n_{i}^{1} / n$.

3) Repeat step (2) with $P_{i}^{\mathrm{EPI}} f_{i}^{2}$, etc.

4) Continue until convergence is satisfactory, i.e. $f_{i}^{K} \simeq$ $f_{i}^{K-1}=f_{i}$.

The values obtained by this method (not shown) are in good agreement with those of the Gaussian fit. 


\subsection{Track by track particle identification and estimated contamination}

Using the population fractions $f_{i}(p)$ described by eq. (5) as an a priori probability, and the $P_{i}^{\mathrm{EPI}}\left(\chi^{2}\right)$ corresponding to eq. (1) for each track, the combined probability is defined as

$P_{i}=f_{i}\left(p_{\text {lab }}\right) P_{i}^{\mathrm{EPI}}\left(\chi^{2}\right)$.

The identification is then made for each track by choosing the mass corresponding to the highest value of $P_{i}$. (Tracks with the highest value of $P_{i}^{\mathrm{EPI}}<0.1 \%$ were excluded). The number of tracks identified in this way in each momentum interval is summarized in table 3. It is straight forward to estimate the contamination of the samples selected in this way by the integration of the Gaussian distributions from the point corresponding to equal values of $P_{i}$. The results given in table 3 show that in the region $22<p_{\text {lab }}<60 \mathrm{GeV} / c$ the average $\pi$ contamination in the $\mathrm{K}$ sample is $-10 \%$, and the corresponding $\mathrm{K}$ contamination in the $\pi$ sample is $\sim 13 \%$. This estimate depends on the assumption that the $I / I_{0}$ distributions are truely Gaussian. Any systematic effects could change this estimate. This value, however, is compatible with a rough estimate derived from the pion sample from $\mathrm{K}^{0}$ decay described in sect. 4.3: taking $f_{\pi}=f_{\mathrm{K}}$ for this sample (a most pessimistic assumption) leads to the wrong identification of $-7 \%$ of the tracks. It is of course possible to obtain purer samples, with a loss of statistics, by requiring a ratio of $P_{\text {, values greater }}$ than one for the mass identification.

An alternative approach to the mass identification is to use a continuous weighting method, such that each track enters a given distribution for a specific mass with an identification weight $W_{i}^{1}$, based on the relative heights of the Gaussian probability distributions of the different particle types, multiplied by the population fraction obtained from the global fits [eq. (5)]. Thus

$$
W_{i}^{\prime}=\frac{f_{i} G_{i}}{\sum_{i=\pi . \mathrm{K} . \mathrm{p}} f_{i} G_{i}},
$$

and

$G_{t}=\frac{1}{\sqrt{2 \pi} \Delta} \exp \left\{-\left[\left(I / I_{0}\right)^{\mathrm{M}}-\left(I / I_{0}\right)_{i}^{\mathrm{T}}\right]^{2} /\left(2 \Delta^{2}\right)\right\}$.

with notation as defined in sect. 3.6.

The numbers of $\pi, \mathrm{K}$ and $\mathrm{p}$, obtained in this way (as the sum of the weighted numbers for each type) in the various momentum bins, are given in table 3 and are found to be in good agreement with the values obtained by the choice method.

\subsection{A study of the resolution}

It is interesting to compare the average resolution obtained in the seven momentum intervals studied, with that expected from the EPI calibration. Clearly, the expected resolution deteriorates as the number of clean cells $N$ decreases. It is therefore important to note that for the selected samples of tracks with $N \geqslant 50$ the mean number of cells per track $\langle N\rangle$ varies smoothly from 90.8 for the highest momentum interval to 71.8 for the lowest, and for tracks with $N \geqslant 80\langle N\rangle$ varies from 104.8 to 91.3. Fig. 19 shows the fitted width (expressed as $W^{\mathrm{K}}$ in percent of $I / I_{0}$ ) for the different momentum ranges as a function of $\langle N\rangle$ both for the $N \geqslant 50$ and $N \geqslant 80$ samples. These data points extrapolate smoothly to a value consistent with the calibration value of $6.6 \%$ for $N=128$ (as indicated by the dashed curve). To calculate the expected resolution in a given momentum interval, resolution functions have been generated as the convolution of Gaussian distributions with widths corresponding to the observed $N$ distribution calculated as

Table 3

(a) Number of identified tracks in various momentum intervals. The upper row of each pair corresponds to the probability choice method and the lower to the continuous weighting method.

(b) The estimated contamination in the samples selected by the probability choice method.

\begin{tabular}{|c|c|c|c|c|c|c|c|}
\hline$p_{\text {lab }}(\mathrm{GeV} / c)$ & $10-15$ & $15-22$ & $22-28$ & $28-35$ & $35-45$ & $45-60$ & $>60$ \\
\hline \multirow[t]{2}{*}{ (a) Identified $\pi$ 's } & 214 & 861 & 896 & 690 & 436 & 222 & 283 \\
\hline & 213 & 860 & 884 & 677 & 421 & 222 & 253 \\
\hline \multirow[t]{2}{*}{ Identified K's } & 75 & 569 & 749 & 784 & 732 & 644 & 1042 \\
\hline & 72 & 563 & 756 & 807 & 743 & 637 & 1064 \\
\hline \multirow[t]{2}{*}{ Identified p's } & 25 & 109 & 98 & 104 & 49 & 8 & 0 \\
\hline & 29 & 116 & 102 & 94 & 52 & 14 & 7 \\
\hline \multicolumn{8}{|c|}{ (b) Contamination (\%) } \\
\hline K's in $\pi$ 's & 5 & 7 & 8 & 13 & 17 & 26 & 28 \\
\hline$\pi$ 's in K's & 12 & 10 & 9 & 11 & 10 & 8 & 6 \\
\hline
\end{tabular}






Fig. 19. Fitted fractional width (fwhm) of Gaussian distribution for kaons $W^{\mathrm{K}}$, as a function of $\langle N\rangle$ for the six momentum intervals indicated for tracks with $N \geqslant 50$ (open points with error bars) and $N \geqslant 80$ (solid points with error bars). The points without error bars are the corresponding widths of the generated resolution functions. The calibration value of $W_{128}^{\mathrm{K}}=$ $6.6 \%$ is indicated by " $\mathrm{x}$ ". The solid curve decreases as $\langle N\rangle^{-0.45}$, the dashed curve is the parametrization (6).

$W_{N}=6.6 \%(N / 128)^{-0.45}$ [statistical term in eq. (4)]. The fractional widths of these resolution functions are also shown in fig. 19 as a function of $\langle N\rangle$. The widths of the resolution functions follow closely the dependence $\alpha\langle N\rangle^{-0.45}$. From this figure it is seen that the fitted experimental resolution lies systematically above the resolution expected from the purely statistical $N$ dependence from fig. 13, but the discrepancy becomes less with samples of increasing $\langle N\rangle$, ranging from $\sim 1.25$ with $\langle N\rangle \sim 70$ to 1.04 with $\langle N\rangle \sim 105$.

This dependence of the width $W$, is parametrized as

$W_{N}=6.6 \%\left(\frac{128}{N}\right)^{0.45}+2.6 \%\left(\frac{128-N}{N}\right)$,

where the second term is the expression for $w$ introduced in eq. (4) to take into account the factors other than the purely statistical that lead to broadening of the $I / I_{0}$ distributions as $\langle N\rangle$ decreases. The tracks with lower values of $N$ arise if the track enters the EPI at a large angle to the EPI axis, thus not traversing the full EPI, or when tracks are heavily cleaned due to the presence of considerable backgrounds. Here systematic effects tend to broaden the ionization distributions.

For the highest momentum interval fitted (60-70 $\mathrm{GeV} / \mathrm{c}$ ), not included in fig. 19, there is a further deterioration of resolution observed both in the $N \geqslant 50$ and $N \geqslant 80$ samples due to variations of response of the

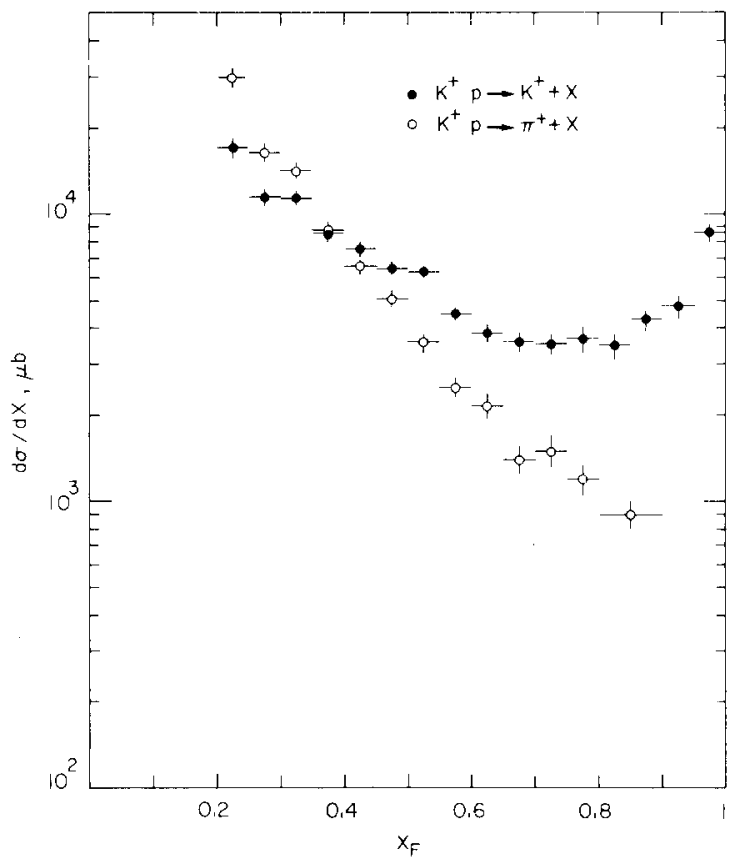

Fig. 20. Differential cross sections $\mathrm{d} \sigma / \mathrm{d} x_{\mathrm{F}}$ for the reactions $\mathrm{K}^{+} \mathrm{p} \rightarrow \pi^{+}+X$ and $\mathrm{K}^{+} \mathrm{p} \rightarrow \mathrm{K}^{+}+X$ obtained using the EPI data.

EPI in the region close to the beam hole, and background beam tracks giving rise to resolutions $\sim 25-30 \%$ larger than statistically predicted for that $N$ cell distribution.

\subsection{An example of physics results for $K^{+} p$ interactions}

The use of EPI information for investigations of the physics of $\mathrm{K}^{ \pm} \mathrm{p}$ interactions at high energies is in progress. As an example, in fig. 20 we present the differential cross sections $\mathrm{d} \sigma / \mathrm{d} x_{\mathrm{F}}$ (where $x_{\mathrm{F}}=2 p_{\|}^{*} / \sqrt{s}$ with $p_{\|}^{*}$ being the longitudinal c.m. momentum and $\sqrt{s}$ the c.m. energy) for the inclusive reactions (elastic scattering events removed)

$\mathrm{K}^{+} \mathrm{p} \rightarrow \pi^{+}+$anything,

$\mathrm{K}^{+} \mathrm{p} \rightarrow \mathrm{K}^{+}+$anything.

The identification of the $\pi^{+}$and $\mathrm{K}^{+}$has been done using the continuous weighting method described above with the acceptance and efficiency corrections applied. The interpretation of these results is outside the scope of the present paper but is discussed in ref. 9 .

\section{Conclusions}

The relativistic rise of ionization has been used in actual physics experiments for the identification of sec- 
ondary particles arising from $\mathrm{K}^{ \pm} \mathrm{p}$ interactions at 70 and $110 \mathrm{GeV} / c$. Using the $\mathrm{BEBC}$ bubble chamber together with the External Particle Identifier, the momentum-dependent populations of pions, kaons and protons were determined. Track by track identification yielded samples of kaons and pions with a residual contamination of $\leqslant 12 \%$.

Among the steps needed to reach the required measurement accuracy were a cell-by-cell calibration of the full EPI matrix with a pencil ray beam, an automatic gain control to compensate for the variations caused by changes in atmospheric pressure, and the monitoring of the ionization of the beam particles to correct for a small residual long-term gain drift. In the presence of background due to other beam tracks and to their interactions in the windows, shields etc., methods of pattern recognition and of local cleaning were developed in order to eliminate the cells hit by more than one track.

This elimination leads to a reduction of the number of ionization samples $N$ used for a given track. A study of the resolution of the EPI in these experimental conditions shows that the resolution falls off somewhat faster with decreasing $N$ than expected on purely statistical grounds, due to effects such as difficulties in removing background. The resulting resolution values are in the range $7.6 \%$ to $11 \% \mathrm{fwhm}$, but extrapolate to a value consistent with the $6.6 \%$ fwhm obtained during calibration using single $50 \mathrm{GeV} / c$ pions traversing the full device.
The authors are grateful to the operating crews of the accelerators, the bubble chamber and the hadron beam line for their efforts. They express their gratitude to their teams of bubble chamber scanning and measurement, and particularly to the Bologna HPD Group which provided special measurements at an early stage of the analysis. Finally we wish to thank our colleagues in the EF Division and from the WA27 and WA28 Collaborations for helping to obtain the data and for many useful discussions.

\section{References}

[1] D. Jeanne et al., Nucl. Instr. and Meth. 111 (1973) 287.

[2] M. Aderholz et al, Nucl. Instr. and Meth. 118 (1974) 419

[3] M. Aderholz et al., Nucl. Instr. and Meth. 123 (1975) 237.

[4] P. Lazeyras et al,, IEEE Trans. Nucl. Sci. Vol. NS-26. (1979) 89.

[5] I. Lehraus et al., Nucl. Instr. and Meth. 153 (1978) 347.

[6] G. Ransone et al., Nucl. Phys. Bl67 (1980) 285.

[7] M. Barth et al., Z. Physik C2 (1979) 285.

[8] P.R.S. Wright, Ph.D. Thesis, University of London (1980); V. Baruzzi et al, Int. Conf. on Instrumentation for colliding beam physics. Stanford (February 1982).

[9] M. Spyropoulou-Stassinaki. 12th Int. Symp. on Multiparticle dynamics, Notre-Dame (1981) and CERN/EP 81-122 (1981).

[10] A. Bézaguet et al., CERN BEBC Users' Handbook (1977).

[11] I. Lehraus, CERN/EF/BEAM 76-3 (1976).

[12] F. Bruyant et al.. HYDRA Application Library, Long Write-up GEOPAM, CERN (1974).

[13] H. Klein and L. Pape. HYDRA Application Library, Long Write-up THIRA and USBEMI, CERN (1976). 